\title{
Sistemas geotérmicos mejorados: revisión y análisis de casos de estudio
}

\author{
Maria Alejandra Palacio-Villa ${ }^{1}$, Daniela Blessent ${ }^{1}$, Jacqueline López-Sánchez ${ }^{1}$, David Moreno ${ }^{1 *}$ \\ doi: http://dx.doi.org/10.18273/revbol.v42n1-2020006 @(c) (i)
}

Forma de citar: Palacio-Villa, M.A., Blessent, D., López-Sánchez, J., y Moreno, D. (2020). Sistemas geotérmicos mejorados: revisión y análisis de casos de estudio. Boletín de Geología, 42(1), 101-118. doi: 10.18273/revbol. v42n1-2020006.

\section{RESUMEN}

En este artículo se hace una revisión bibliográfica de las características de los EGS, fuentes de energía limpia que prometen ser una alternativa para enfrentar los problemas relacionados con el calentamiento global ocasionados por el uso de combustibles fósiles como el petróleo y el gas natural. Actualmente en Colombia los sistemas geotérmicos de interés son de tipo hidrotermal, por lo que no hay EGS planificados aún, sin embargo, este artículo pretende ser una introducción para el lector interesado en los EGS y ser referencia a futuros proyectos desarrollados en el territorio nacional, describiendo los lugares del mundo más significativos donde se ha hecho uso de esta técnica, junto con su percepción social e impactos asociados. Además, busca analizar las diferencias entre la estimulación hidráulica en los EGS y el fracking utilizado para la extracción de gas de esquisto.

Palabras clave: Sistemas Geotérmicos Mejorados (EGS); Fracking; Impactos ambientales; Energía Geotérmica; Energía renovable.

\section{Enhanced geothermal systems: review and analysis of case studies}

\begin{abstract}
This article reviews the characteristics of the EGS, sources of clean energy that promise to be an alternative to face the problems related to global warming caused by the use of fossil fuels like oil and natural gas. Currently in Colombia the geothermal systems of interest are hydrothermal, so there are no plans for the development of EGS yet, however, this article pretends to be an introduction to the reader interested in EGS and to be a reference to future projects developed in the national territory, describing the most significant places in the world where this technique has been used, along with its social perception and associated impacts. In addition, it seeks to analyze the differences between the hydraulic stimulation technique in the EGS and the fracking used for the extraction of shale gas.
\end{abstract}

Keywords: Enhanced Geothermal Systems (EGS); Fracking; Environmental Impacts; Geothermal Energy; Renewable Energy.

1Programa Ingeniería Ambiental, Universidad de Medellín, Medellín, Colombia. malejandrapalaciov@gmail.com, dblessent@udem.edu.co,ilopez@udem.edu.co, (*) damoreno@udem.edu.co 


\section{INTRODUCCIÓN}

Los combustibles fósiles como el carbón, el petróleo y el gas natural, principales fuentes de producción de energía, son considerados generadores de emisiones de partículas y gases contaminantes a la atmosfera que favorecen el efecto invernadero (Gurney et al., 2009; BP, 2019; REN21, 2019). La modificación de los patrones de precipitación, el deshielo de los glaciares, el aumento en el nivel del mar, los cambios en las corrientes oceánicas, la acidificación de las aguas, y la ocurrencia de tormentas más poderosas y de mayor duración son algunos de los impactos generalmente asociados al fenómeno, relacionados con el aumento en la temperatura del océano y la atmosfera terrestre (Conde-Álvarez y Saldaña-Zorrilla, 2007).

Para mitigar estos impactos, las energías renovables son una solución eficiente y efectiva (Dincer, 2000), son consideradas alternativas prometedoras y limpias a nivel mundial ya que son producidas a partir de fuentes naturales no sujetas al agotamiento, como el sol, el viento, las olas, las mareas, la energía potencial del agua y el calor de la Tierra; además son consideradas neutras en carbono, liberando relativamente pocas emisiones a la atmosfera (Elum y Momodu, 2017).

La energía geotérmica, asociada al calor terrestre, tiene múltiples formas de aprovechamiento, entre las que se destacan la generación de electricidad (ChamorroCamazón, 2009), la calefacción o enfriamiento en los edificios (Ruíz-Calvo, 2015), el secado de productos agrícolas y la calefacción de invernaderos, entre otros (Popovska-Vasilevska, 2003). Su utilización para producción de electricidad se ha limitado históricamente a sitios específicos, ya que para hacer posible el aprovechamiento convencional de este recurso es indispensable encontrar una zona de la corteza terrestre con las siguientes características naturales (DiPippo, 2012), que se muestran en la Figura 1:

1. Una gran fuente de calor a una profundidad técnica y económicamente viable

2. Un reservorio permeable

3. Suficiente suministro de fluido geotérmico (agua al estado líquido o vapor)

4. Una capa sello superior (roca impermeable)

5. Un mecanismo de recarga confiable

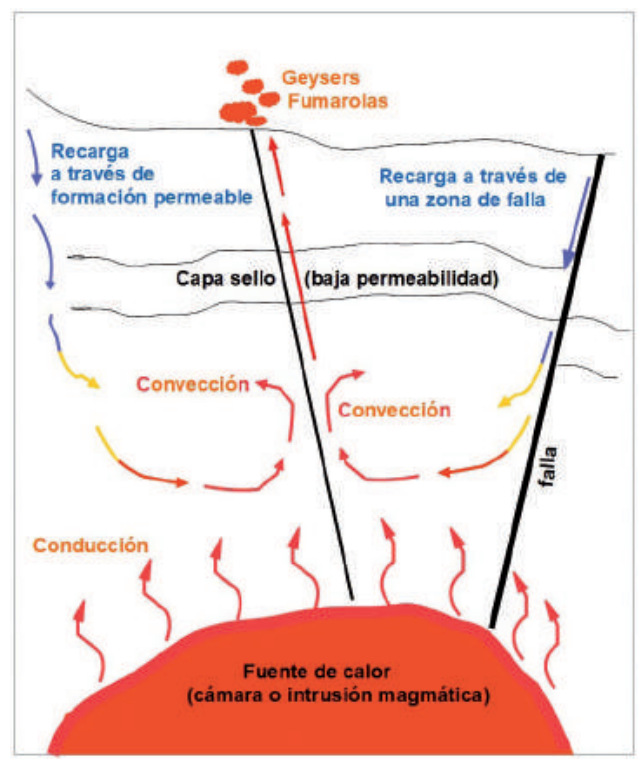

Figura 1. Esquema conceptual que muestra los principales elementos de un sistema geotérmico.

Las zonas en la corteza terrestre que cumplen con estos cinco requerimientos se denominan yacimientos geotérmicos, y pueden clasificarse según su temperatura como: yacimiento de baja entalpia $(<$ $\left.90^{\circ} \mathrm{C}\right)$, media entalpia $\left(90^{\circ} \mathrm{C}-150^{\circ} \mathrm{C}\right)$ y alta entalpia $(>$ $\left.150^{\circ} \mathrm{C}\right)$ (Hochstein, 1988).
En la actualidad no todas estas condiciones deben cumplirse para el aprovechamiento energético del calor, ya que, para la producción de electricidad, la necesidad de una alta temperatura puede compensarse con una mayor profundidad de perforación y la ausencia del agua circulante en el reservorio puede 
solucionarse con su inyección desde la superficie (Chamorro-Camazón, 2009). Ha sido entonces posible que el aprovechamiento del calor se concrete, gracias a la implementación de técnicas que permiten asegurar la permeabilidad y circulación de fluidos a través de los Sistemas Geotérmicos Mejorados (EGS de sus siglas en inglés) (Bendall et al., 2014). Esta tecnología ha sido desarrollada desde el año 1970 con el concepto HDR (Hot Dry Rock) (Tenzer, 2001), lo que permite garantizar la viabilidad en la extracción de calor y generación de energía mediante la perforación a grandes profundidades y creación de fracturas en la formación geológica impermeable (Brace, 1980). Para esto, se requiere la ayuda de procesos de fracturación hidráulica, un proceso que busca generar fracturas en la roca, y/o aumentar el tamaño, la extensión y la conectividad de las existentes (Burton et al., 2014). Esta práctica es utilizada para la optimización de la productividad en pozos donde la alta temperatura y la permeabilidad se dan simultáneamente (Olasolo, 2014), y para la estimulación donde los recursos hidrotermales no se encuentran presentes (McClure y Horne, 2014). Las principales etapas de desarrollo de un sistema EGS se muestran esquemáticamente en la Figura 2.
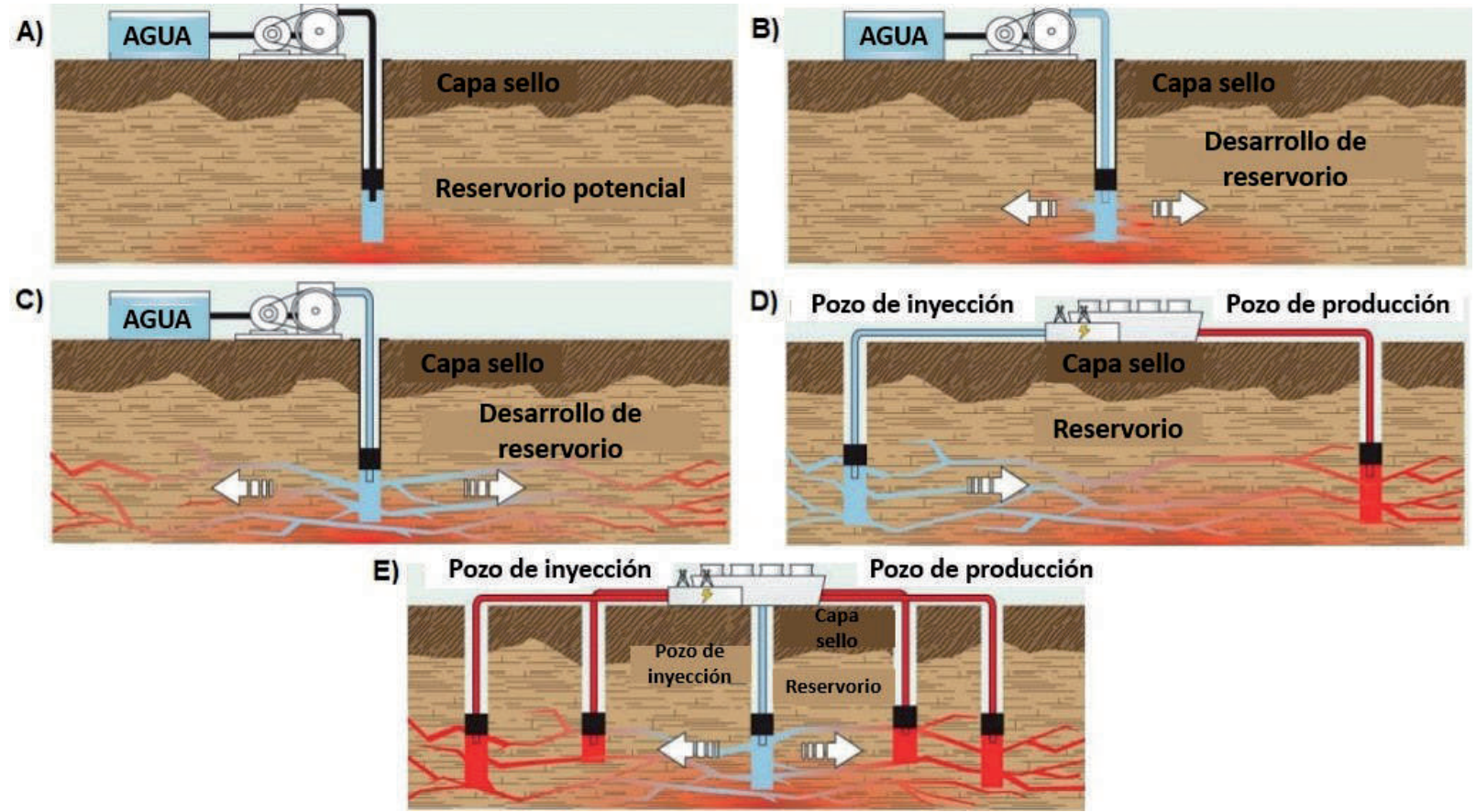

Figura 2. Etapas de un EGS. A. Un pozo de inyección es perforado en un basamento de alta temperatura, de baja permeabilidad y con poca cantidad de fluidos. B. Se inyecta agua con suficiente presión para asegurar un nuevo fracturamiento o la apertura de las fracturas existentes. C. Se continúa el bombeo de agua al interior del reservorio para expandir las fracturas. D. Un pozo de producción es perforado con la intención de interceptar el sistema de fracturas para extraer el calor del yacimiento estimulado. E. Pozos de producción adicionales son perforados para extraer grandes volúmenes de calor y utilizar estos recursos para la generación eléctrica, entre otros usos (tomado y modificado de U.S. Department of Energy, 2004).

La implementación de cualquier tecnología tiene complejidades técnicas e impactos asociados, en los EGS, los desafíos ambientales están relacionados con el consumo de agua (Clark et al., 2009) y la sismicidad inducida (Lacirignola y Blan, 2013). Estos impactos deben estudiarse detalladamente con el fin de entender su proceso, las posibles soluciones o alternativas a estos impactos y el beneficio que presenta el uso de estas técnicas en reemplazo de los combustibles convencionales. A través de este artículo se pretende ofrecer al lector una introducción sobre dichos aspectos, gracias a la revisión bibliográfica realizada.

\section{PRINCIPALES PROYECTOS EGS EN EL MUNDO}

Las características principales de los EGS considerados más representativos y analizados en este trabajo están resumidas en la Tabla 1 y su localización puede observarse en la Figura 3. Los sitios descritos son Soultz-sous-Forêts en Francia, Basilea en Suiza, Groß Schönebeck en Alemania, Habanero en Australia y el proyecto Fallon FORGE en Estados Unidos. 


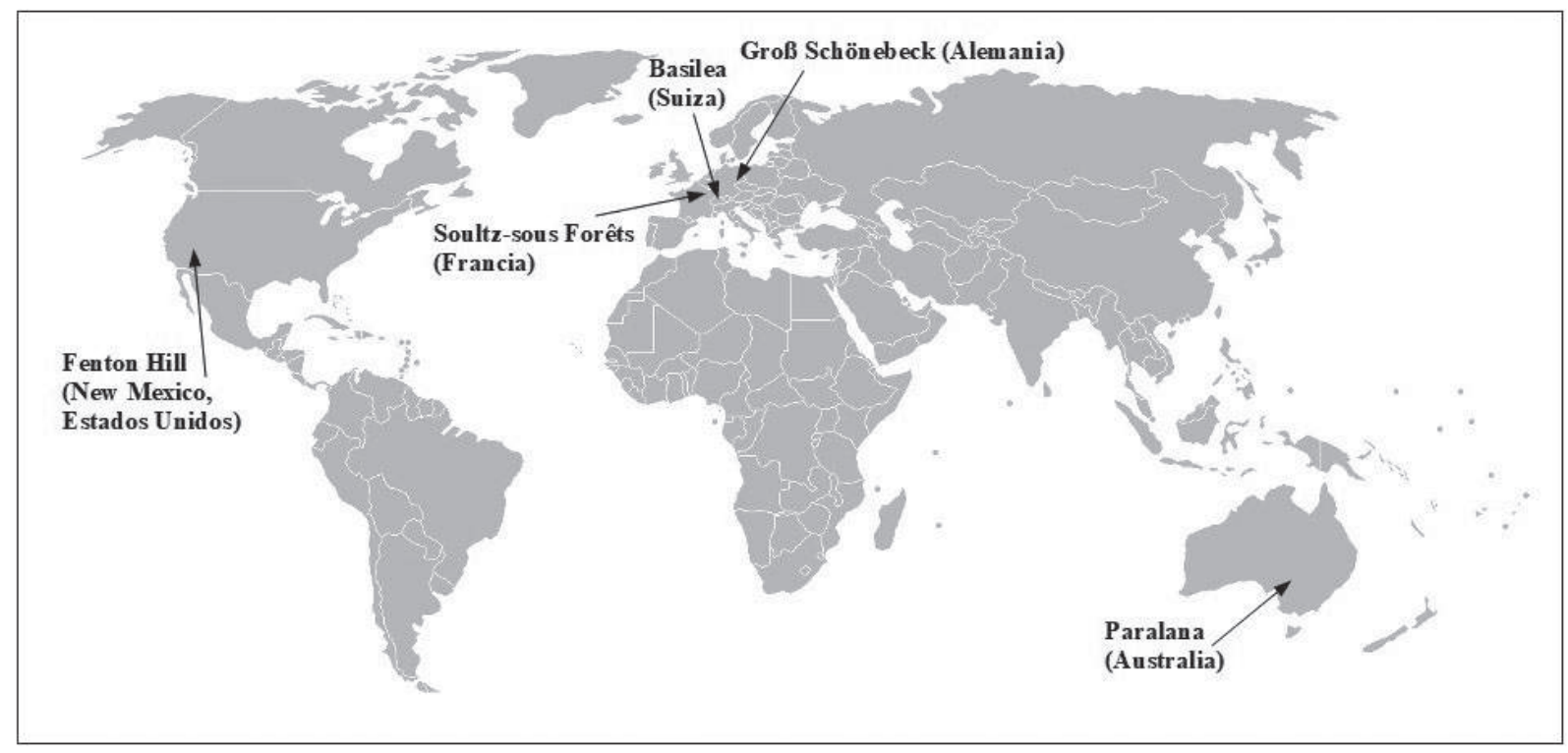

Figura 3. Ubicación de los sistemas EGS analizados en este trabajo.

Otros sitios en los cuales se han realizados algunos estudios e investigaciones son Ogachi (Japón), New Berry (Estados Unidos) y Rosemanowes (Reino Unido). El proyecto Ogachi fue llevado a cabo por el Instituto Central de Investigación de la Industria de Energía Eléctrica (CRIEPI), situado en el sur de Akita, Japón (Kaieda, 2012). De 1989 a 2002 se crearon dos reservorios a diferentes profundidades desde un pozo de inyección de $1000 \mathrm{~m}$ de profundidad y se perforaron dos pozos de producción para penetrar en los reservorios (Kaieda et al., 2005). El proyecto Newberry, ubicado en el flanco occidental del volcán Newberry en el centro de Oregón, Estados Unidos, tiene como objetivo mejorar la viabilidad de las tecnologías y aplicaciones de EGS (Cladouhos et al., 2018). El proyecto HDR Rosemanowes fue realizado de 1983 a 1991 cerca de Penryn en Cornwall, Inglaterra. Se perforaron tres pozos y se llevaron a cabo varios experimentos de estimulación (DECC, 2013). Los yacimientos creados obtuvieron profundidades de hasta $2500 \mathrm{~m}$.

\section{Fenton Hill (Estados Unidos)}

El primer acercamiento a los EGS, tiene su origen en el laboratorio nacional de Los Álamos (LANL), ciudad de Nuevo México, en Estados Unidos, con el proyecto Fenton Hill (Olasolo et al., 2016) que fue llevado a cabo entre los años de 1974 a 1995 (Xia et al., 2017). El área fue seleccionada porque las rocas precámbricas cristalinas, formación objetivo, son térmicamente aisladas por depósitos sedimentarios paleozoicos de grano fino y de gran espesor, a su vez recubiertos por lava y toba volcánica de flujos de ceniza del Campo Volcánico de Jemez, ubicado a 15-40 km de LANL (Kelkar et al., 2016). Las investigaciones y trabajo de campo fueron desarrollados en un yacimiento de $3000 \mathrm{~m}$ de profundidad con una temperatura de aproximadamente $200^{\circ} \mathrm{C}$, donde 13 perforaciones permitieron ubicar un área de alto flujo de calor (Lu, 2018). El pozo GT-1 fue perforado a $785 \mathrm{~m}$ de profundidad y diseñado para medir el flujo de calor en el sitio, adquirir muestras de rocas y probar técnicas de fracturación hidráulica (Reiter et al., 1976). En 1979 la perforación del pozo EE2 alcanzó un depósito más profundo $(4400 \mathrm{~m})$ y más caliente $\left(300^{\circ} \mathrm{C}\right)($ Kelkar et al., 2016).

Aunque este lugar fue cerrado debido a que no alcanzó la capacidad de producción esperada (Tester et al., 2006), logró el mejoramiento de la tecnología para la extracción de calor (Barbier, 2002) y suministró resultados importantes, como la verificación de la viabilidad técnica de la perforación a $5000 \mathrm{~m}$ de profundidad y la confirmación de que la fracturación hidráulica se puede utilizar para incrementar el aprovechamiento del calor (Lu, 2018). La conclusión más importante fue que un sistema EGS se puede formar a cualquier profundidad, con temperatura y conectividad suficiente a través de las fracturas creadas o estimuladas (Brown, 2009). Beneficiándose de la experiencia y el interés generado por el proyecto pionero, otros países experimentaron con el desarrollo del concepto de crear un reservorio geotérmico en otros entornos geológicos (Tester et al., 2006). 


\section{Soultz-Sous-Forêts (Francia)}

La zona de Soultz se caracteriza por un gradiente excepcionalmente alto cerca de la superficie, alrededor de $110^{\circ} \mathrm{C} / \mathrm{km}$, aproximadamente tres veces más alto que lo normal (DiPippo, 2012).

Este proyecto es el resultado de 20 años de investigación activa basada en la geología, geoquímica, geofísica, hidráulica y modelación: estos estudios y el uso de buenas técnicas permitieron la instauración de una planta piloto, siendo la primera en el mundo de este tipo en producir electricidad (Bertani, 2012). La zona geotérmica Soultz se encuentra en el Graben del Alto Rin, que forma parte del sistema europeo de rift cenozoico que se extiende desde el Mediterráneo hasta la costa del Mar del Norte. En Soultz, el basamento granítico fallado está cubierto por $1400 \mathrm{~m}$ de sedimentos (Genter et al., 2010).

En la primera fase del proyecto, se realizó la exploración y estimulación a una profundidad de $2000 \mathrm{~m}$ (Cuenot et al., 2008). Con los resultados obtenidos, se procedió a la perforación de 3 pozos a una profundidad aproximada de $5000 \mathrm{~m}$, alcanzando temperaturas de $200^{\circ} \mathrm{C}$ (Dubois et al., 1996). Dos de los pozos son de producción y se usan para bombear agua que se reinyecta a una menor temperatura en el tercer pozo de inyección (Cuenot et al., 2008). Entre
2004 y 2009 se construyó la planta geotérmica binaria con ciclo orgánico de Rankine que utiliza un fluido de trabajo de baja ebullición (isobutano). Un esquema de los principales elementos de una central binaria se presenta en la Figura 4, donde se puede observar que el fluido extraído por el pozo de producción pasa por el sistema de precalentador y evaporador (intercambiador de calor) y es enviado directamente al pozo de reinyección, sin que entre en contacto con la turbina ni con la atmosfera. De hecho, el fluido geotérmico en este sitio es altamente salino, lo que no permite su vaporización directa en la turbina ni la temperatura de producción esperada (Genter et al., 2010), por lo que se requiere utilizar un fluido de trabajo. El geofluido es una salmuera de cloruro de sodio con aproximadamente 100.000 ppm de sólidos totales disueltos, un $\mathrm{pH}$ de 4,9 y una temperatura de $200^{\circ} \mathrm{C}$; cuando llega a la planta, la temperatura desciende a $175^{\circ} \mathrm{C}$ (DiPippo, 2012). La central se caracteriza por una capacidad de 1,5 MW de potencia eléctrica.

La estimulación hidráulica fue la principal técnica utilizada para aumentar la permeabilidad del reservorio (Schill et al., 2017). Este proyecto es un ejemplo de estimulaciones exitosas, mostrando la importancia de estos tratamientos. La planta de energía en Soultz se abrió en 2008, con un caudal de 35 1/s y una temperatura de producción de $175^{\circ} \mathrm{C}$ (Schindler et al., 2010).

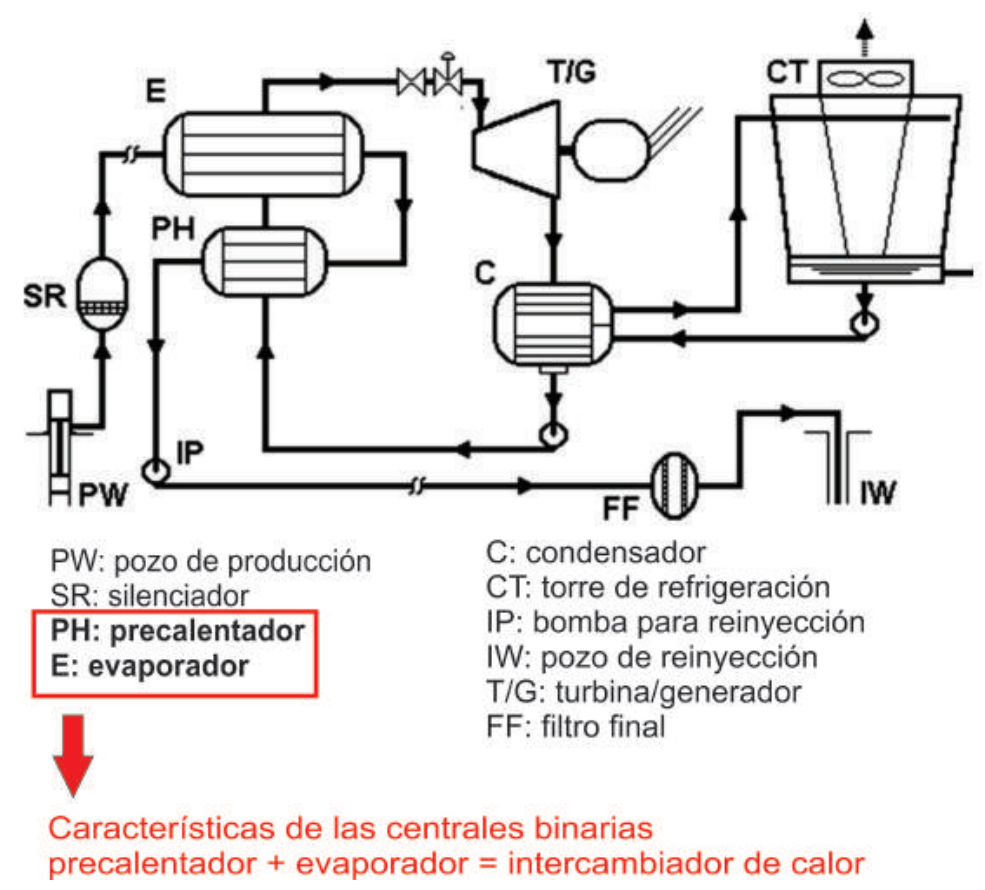

Figura 4. Esquema simplificado de una central geotérmica binaria con ciclo orgánico de Rankine (tomado y modificado de DiPippo, 2012). 


\section{Basilea (Suiza)}

El proyecto Basilea, ubicado en la misma ciudad suiza, tuvo como objetivo crear plantas de generación eléctrica a nivel comercial ( $\mathrm{Lu}, 2018)$. Fue un proyecto EGS liderado por la empresa Geopower Basel AG que durante el 2006 llevó a cabo una prueba de estimulación hidráulica a una profundidad aproximada de 5000 m (Wyss y Rybach, 2010) en un basamento granítico $(2600 \mathrm{~m})$, recubierto por $2400 \mathrm{~m}$ de sedimentos del Cuaternario, Terciario, Mesozoico y Pérmico y alrededor de $100 \mathrm{~m}$ de limolita roja y granito alterado; el basamento es la continuación meridional del macizo montañoso de la Selva Negra, constituido únicamente por rocas plutónicas, sin presencia de rocas metamórficas (Häring et al., 2008).

Antes de perforar el pozo Basel-1 de $5009 \mathrm{~m}$ de profundidad, donde se llevó a cabo la estimulación en correspondencia del basamento cristalino granítico, se perforó un pozo de reconocimiento a $2755 \mathrm{~m}$ y se instaló un sistema de monitoreo microsísmico con cinco pozos menos profundos con distancias entre 300 y $2750 \mathrm{~m}$. Este sistema de monitoreo no solo se instaló para conocer la actividad microsísmica que podría tomar lugar durante la estimulación, sino para observar de igual manera la sismicidad natural del sitio (Häring et al., 2008). Las mediciones de temperatura se adquirieron poco después de alcanzar la profundidad final, cuando la temperatura del reservorio todavía estaba perturbada por la perforación: diferentes métodos de extrapolación permitieron estimar una temperatura del reservorio de $190^{\circ} \mathrm{C}$ a $5000 \mathrm{~m}$ de profundidad (Häring et al., 2008). La prueba de estimulación fue detenida ya que ocasionó problemas de microsismicidad con un sismo de magnitud 3.4 (Häring et al., 2008), causando daños estructurales en el sitio y generando miedo en la población, por lo que el proyecto se interrumpió (Lu, 2018). Gracias a lo sucedido, la Comisión Europea cofundó junto con la coordinación del Centro Internacional de Investigación Geotérmica (GEISER), un proyecto para regular los EGS integrando la mitigación de la sismicidad inducida en los reservorios (Zang et al., 2014).

El proyecto esperaba ser una de las primeras plantas de energía comercial basadas en la tecnología EGS (Häring et al., 2008). Los primeros micro sismos fueron detectados en el 2006, donde se produjeron más de 160 eventos de los cuales solo cuatro pudieron ser ubicados y ocurrieron cuando el agua era bombeada fuera del pozo para reducir la presión (Kraft et al., 2009). Todos los sismos localizables ocurrieron en una distancia de menos de 100 metros del pozo de inyección, a una profundidad aproximada de $4400 \mathrm{~m}$, donde en análisis posteriores se detectó la zona de infiltración del reservorio (Häring et al., 2008). De este proyecto se pueden determinar los criterios de selección de los sitios para la implementación de los sistemas EGS: evitar áreas densamente pobladas, zonas con actividad sísmica natural media-alta, y que se encuentren a una distancia de al menos un kilómetro de distancia de las zonas de fallas regionales o principales (Meier et al., 2015).

\section{Groß Schönebeck (Alemania)}

La plataforma de investigación geotérmica GFZ Groß Schönebeck está situada al noreste de Berlín, en el extremo sur de la cuenca del norte de Alemania ( $\mathrm{Lu}$, 2018). Con el fin de generar electricidad geotérmica a partir de yacimientos de baja entalpía el reservorio de la cuenca fue elegido para desarrollar la técnica EGS (Huenges et al., 2006). El reservorio profundo consiste en una secuencia de areniscas, conglomerados y rocas volcánicas con fluidos a $150^{\circ} \mathrm{C}$ y porosidades hasta el $10 \%$, recubiertos por $2370 \mathrm{~m}$ de sedimentos cuaternarios a triásicos y $1492 \mathrm{~m}$ de sales de Zechstein (ENGINE, 2008).

En este sistema, dos pozos de exploración forman un doblete geotérmico, donde se usa un pozo para la extracción del fluido y otro para su reinyección una vez el calor ha sido extraído. Se explotan acuíferos a profundidades entre 3900 y $4400 \mathrm{~m}$ con temperaturas de hasta $150^{\circ} \mathrm{C}$ (Min et al., 2015). El pozo denominado Groß Schönebeck 3/90, originalmente terminado en 1990, fue perforado de nuevo en 2000, estimulado hidráulicamente en 2002 y 2003, y probado en 2003, 2004 y 2005. En abril de 2006, comenzó la perforación en un segundo pozo para mejorar el flujo másico (Huenges et al., 2006).

Este proyecto se realizó con el fin de establecer un laboratorio in-situ para desarrollar métodos de estimulación adecuados para aumentar la permeabilidad (Blöcher et al., 2015).

\section{Paralana (Australia)}

Este proyecto fue desarrollado por la empresa Petratherm Limited, ubicado $600 \mathrm{~km}$ al norte de la ciudad de Adelaida, en el sur de Australia (Bendall et al., 2014). Petratherm Limited es una empresa que inicialmente buscaba construir una planta de energía geotérmica con una producción entre 3,75 - 7,5 MW de potencia eléctrica (King et al., 2009). El proyecto 
Paralana explora los recursos viables en el graben de Poontana, donde las rocas del basamento están recubiertas por una espesa secuencia de sedimentos neoproterozoicos, cámbricos y terciarios.

Se inició con las actividades de exploración en la región con la perforación de un pozo de prueba (Paralana 1B) a 491m. En junio de 2006, se profundizó el pozo Paralana 1B a $1807 \mathrm{~m}$ para evaluar los gradientes de temperatura de los estratos geológicos más profundos, en donde se registró una temperatura de $109^{\circ} \mathrm{C}$ y un flujo de calor calculado de $129 \mathrm{~mW} / \mathrm{m}^{2}$ (Reid et al., 2012). En la segunda mitad de 2009, un pozo geotérmico, Paralana 2, fue diseñado para ser un pozo inyector y perforado a $4003 \mathrm{~m}$ de profundidad (Bendall et al., 2014). Se realizaron operaciones de estimulación hidráulica en 2011. En 2014 se programó la perforación de un tercer pozo, incluyendo la fracturación hidráulica y la circulación de fluidos y en 2015 se programó la construcción de una planta de generación eléctrica de ciclo binario de $3.5 \mathrm{MW}$ de potencia eléctrica (Lu, 2018).

\section{Fallon Forge (Estados Unidos)}

El proyecto Fallon FORGE del Observatorio de la Frontera para la Investigación en Energía Geotérmica (FORGE) se encuentra ubicado dentro de la Estación Naval Aérea Fallon al sureste de la ciudad de Fallon, Nevada, en Estados Unidos (Robertson-Tait et al., 2018). La zona se ubica en rocas metamórficas y graníticas de baja permeabilidad y temperaturas mayores de $175^{\circ} \mathrm{C}$, aproximadamente a $1700 \mathrm{~m}$ de profundidad (Siler et al., 2018). Varios eventos tectónicos afectan a esta región y son relevantes para la geología y la estructura del sitio Fallon que se encuentra en una región sísmicamente activa, pero no se ha producido una sismicidad histórica significativa en el sitio, ni ninguna falla cuaternaria corta unidades de superficial en el área de estudio.

El propósito con el que se realizó este proyecto es el establecimiento y administración del laboratorio de investigación, para que las diferentes comunidades interesadas, como la científica e ingenieril puedan desarrollar y probar diferentes tecnologías o técnicas para el mejoramiento de los EGS (Faulds et al., 2015). La fase 1 del proyecto fue enfocada en desarrollar un modelo geológico conceptual y planear las actividades posteriores. Durante la fase 2, se desarrollaron planes detallados para los experimentos. Las fases $2 \mathrm{C}$ y 3 comenzaron a desarrollarse en el 2018 (Ayling et al., 2018). Se anticipa que se perforarán múltiples pozos profundos en el sitio, a profundidades que van desde 1500 hasta $2000 \mathrm{~m}$. Se perforarán pozos poco profundos para monitorear actividades subsuperficiales. En apoyo del trabajo para la fase 3 , el equipo está trabajando actualmente con la Oficina de Campo de Stillwater de la Oficina de Administración de Tierra y Naval Air Station Fallon para preparar la documentación ambiental necesaria y recopilar datos para lograr caracterizar el sitio más detalladamente y reducir la incertidumbre geológica (Blankenship et al., 2017).

Tabla 1. Resumen de los principales proyectos EGS en el mundo.

\begin{tabular}{|c|c|c|c|c|c|}
\hline $\begin{array}{c}\text { Nombre del } \\
\text { proyecto EGS }\end{array}$ & Año & $\begin{array}{l}\text { Litología } \\
\text { donde se } \\
\text { realizó } \\
\text { estimulación } \\
\end{array}$ & $\begin{array}{c}\text { Problemas } \\
\text { asociados con } \\
\text { la sismicidad }\end{array}$ & $\begin{array}{l}\text { Temperatura } \\
\text { registrada }\end{array}$ & Profundidad y número de Pozos \\
\hline \multirow{8}{*}{$\begin{array}{l}\text { Fenton Hill } \\
\text { (Estados } \\
\text { Unidos) }\end{array}$} & \multirow{8}{*}{ 1974-1995 } & \multirow{8}{*}{ Granito } & \multirow{8}{*}{ Sí } & \multirow{8}{*}{$\begin{array}{c}200^{\circ} \mathrm{C} \\
\text { (a } 3000 \mathrm{~m})\end{array}$} & $\begin{array}{l}\text { GT-1 }(785 \mathrm{~m}) \\
\text { Primer pozo usado para medir el flujo calor en el } \\
\text { sitio, adquirir muestras del basamento rocoso, y para } \\
\text { probar la fracturación hidráulica }\end{array}$ \\
\hline & & & & & $\begin{array}{l}\text { EE-1 }(2930 \mathrm{~m}) \\
\text { Pozo de inyección (Fase } 1)\end{array}$ \\
\hline & & & & & $\begin{array}{l}\text { GT-2B } \\
\text { Pozo de producción (Fase 1) }\end{array}$ \\
\hline & & & & & $\begin{array}{l}\text { EE-2 }(3500 \mathrm{~m}) \\
\text { Pozo de producción (Fase } 2)\end{array}$ \\
\hline & & & & & $\begin{array}{l}\text { EE-3 (3500 m) } \\
\text { Pozo de inyección (Fase 2) }\end{array}$ \\
\hline & & & & & $\begin{array}{l}\text { PC-1 }(663,9 \mathrm{~m}) \\
\text { Pozo de monitoreo de sismicidad }\end{array}$ \\
\hline & & & & & $\begin{array}{l}\text { PC-2 }(663,9 \mathrm{~m}) \\
\text { Pozo de monitorio de sismicidad }\end{array}$ \\
\hline & & & & & $\begin{array}{l}\text { EE-3A }(2900 \mathrm{~m}) \\
\text { Redirección de pozo EE-3 }\end{array}$ \\
\hline
\end{tabular}


Continuación Tabla 1.

\begin{tabular}{|c|c|c|c|c|c|}
\hline $\begin{array}{c}\text { Nombre del } \\
\text { proyecto EGS }\end{array}$ & Año & $\begin{array}{c}\text { Litología } \\
\text { donde se } \\
\text { realizó } \\
\text { estimulación }\end{array}$ & $\begin{array}{l}\text { Problemas } \\
\text { asociados con } \\
\text { la sismicidad }\end{array}$ & $\begin{array}{l}\text { Temperatura } \\
\text { registrada }\end{array}$ & Profundidad y número de Pozos \\
\hline \multirow{5}{*}{$\begin{array}{l}\text { Soultz- } \\
\text { sous Forêts } \\
\text { (Francia) }\end{array}$} & \multirow{5}{*}{ 1987-Presente } & \multirow{5}{*}{ Granito } & \multirow{5}{*}{ Sí } & \multirow{5}{*}{$\begin{array}{c}200^{\circ} \mathrm{C} \\
(\text { a } 5000 \mathrm{~m})\end{array}$} & $\begin{array}{l}\text { GPK } 1(3600 \mathrm{~m}) \\
\text { Primer pozo perforado }\end{array}$ \\
\hline & & & & & $\begin{array}{l}\text { GPK } 2(5100 \mathrm{~m}) \\
\text { Pozo de producción }\end{array}$ \\
\hline & & & & & $\begin{array}{l}\text { GPK } 3(5100 \mathrm{~m}) \\
\text { Pozo de reinyección }\end{array}$ \\
\hline & & & & & $\begin{array}{l}\text { GPK } 4(5260 \mathrm{~m}) \\
\text { Pozo de producción }\end{array}$ \\
\hline & & & & & $\begin{array}{l}\text { EPS } 1(2200 \mathrm{~m}) \\
\text { Pozo de observación sísmica }\end{array}$ \\
\hline \multirow[t]{2}{*}{$\begin{array}{l}\text { Basilea } \\
\text { (Suiza) }\end{array}$} & \multirow[t]{2}{*}{$2005-2006$} & \multirow[t]{2}{*}{ Granito } & \multirow[t]{2}{*}{ Sí } & \multirow[t]{2}{*}{$\begin{array}{c}190^{\circ} \mathrm{C} \\
(\text { a } 5000 \mathrm{~m})\end{array}$} & $\begin{array}{l}\text { Otterbach } 2(2755 \mathrm{~m}) \\
\text { Pozo de reconocimiento } \\
\text { Luego pozo de monitoreo microsísmico }\end{array}$ \\
\hline & & & & & $\begin{array}{l}\text { Pozo Basilea } 1(5000 \mathrm{~m}) \\
\text { Pozo de inyección }\end{array}$ \\
\hline \multirow{2}{*}{$\begin{array}{l}\text { Groß } \\
\text { Schönebeck } \\
\text { (Alemania) }\end{array}$} & \multirow{2}{*}{ 2007-Presente } & \multirow{2}{*}{$\begin{array}{c}\text { Roca } \\
\text { sedimentaria }\end{array}$} & \multirow{2}{*}{ Sí } & \multirow{2}{*}{$\begin{array}{c}150^{\circ} \mathrm{C} \\
(\mathrm{a} 3900-4400 \\
\mathrm{m})\end{array}$} & $\begin{array}{l}\text { Groß Schönebeck 3/90 (4309 m) } \\
\text { Pozo de exploración de gas }\end{array}$ \\
\hline & & & & & $\begin{array}{l}\text { Groß Schönebeck 4/05 (4440 m) } \\
\text { Pozo geotérmico }\end{array}$ \\
\hline \multirow{5}{*}{$\begin{array}{l}\text { Paralana } \\
\text { (Australia) }\end{array}$} & \multirow{5}{*}{$2005-2018$} & \multirow{5}{*}{$\begin{array}{c}\text { Roca } \\
\text { sedimentaria }\end{array}$} & \multirow{5}{*}{ Sí } & \multirow{5}{*}{$\begin{array}{c}109^{\circ} \mathrm{C} \\
\text { (a } 1807 \mathrm{~m})\end{array}$} & $\begin{array}{l}\text { Paralana } 1(500 \mathrm{~m}) \\
\text { Perforación de evaluación superficial }\end{array}$ \\
\hline & & & & & $\begin{array}{l}\text { Paralana 1B }(1807 \mathrm{~m}) \\
\text { Profundización del pozo Paralana }\end{array}$ \\
\hline & & & & & Paralana $2(4003 \mathrm{~m})$ pozo de inyección \\
\hline & & & & & H01 Pozo de inyección \\
\hline & & & & & H02, H03 y H03 pozos de producción \\
\hline $\begin{array}{c}\text { Fallon Forge } \\
\text { (Estados } \\
\text { Unidos) }\end{array}$ & En desarrollo & $\begin{array}{l}\text { Rocas } \\
\text { metamórficas y } \\
\text { granito }\end{array}$ & - & $\begin{array}{c}>175^{\circ} \mathrm{C} \\
\text { (a } 1700 \mathrm{~m})\end{array}$ & Pozos a una profundidad aproximada de $1500-2000 \mathrm{~m}$ \\
\hline
\end{tabular}

\section{DISCUSIÓN}

\section{Aspectos técnicos}

Los EGS son creados mediante la estimulación de rocas de baja permeabilidad (Rongved, 2015). Generalmente, hay tres configuraciones básicas de plantas de energía geotérmica: vapor seco, tipo flash (simple, doble y múltiple), y binario.

Los métodos utilizados para estimular estos sistemas se pueden clasificar como estimulación hidráulica, que incluye fracturamiento y cizallamiento hidráulico, estimulación térmica y estimulación química (Watanabe et al., 2017), como se resume en la Figura 5.
La estimulación hidráulica genera nuevas fracturas o abre fracturas existentes debido a la inyección de fluido a alta presión (Darnet et al., 2006). El cizallamiento hidráulico reactiva las fracturas existentes que se encuentran orientadas favorablemente, el desplazamiento por cizalladura a lo largo de los planos de fractura produce aumento de la permeabilidad (Ucar et al., 2018). La estimulación térmica se basa en la inyección de agua fría en rocas de alta temperatura, causando la contracción térmica de las rocas y mejorando la permeabilidad. Por su parte, la estimulación química es basada en tratamientos ácidos para remover las obstrucciones en las fracturas para mejorar la permeabilidad en las proximidades de los pozos (Xu et al., 2009). 


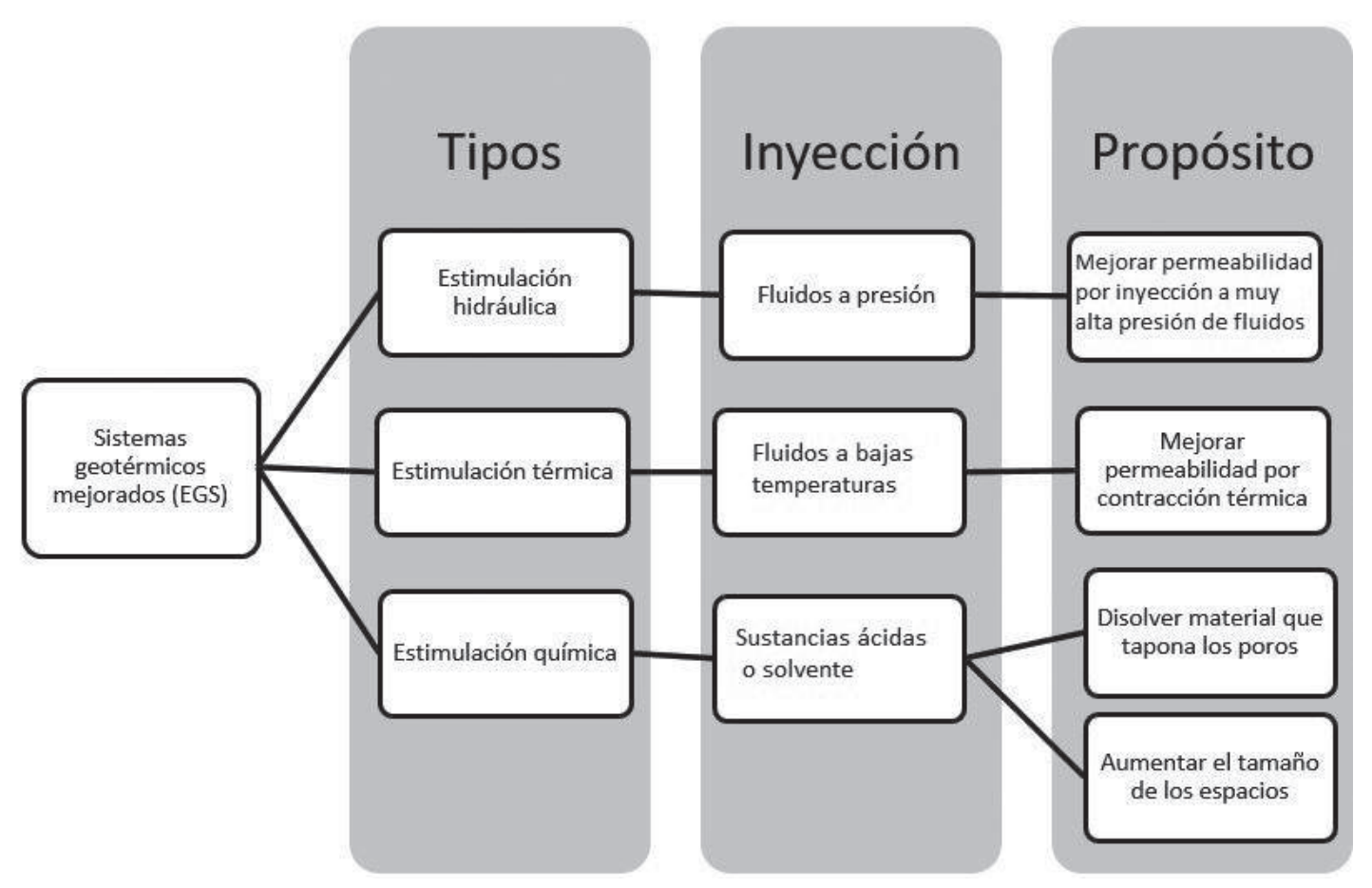

Figura 5. Tipos de estimulación de yacimientos en los EGS.

La estimulación hidráulica tiene una gran relevancia ya que se compara con el proceso realizado en la extracción de gas, contando esta técnica con numerosos detractores y siendo considerada la causante de múltiples impactos sobre el ambiente (Meng y Ashby, 2014). Existe la fracturación de múltiples etapas en pozos horizontales (Guo et al., 2018) y fracturación simultánea (Waters et al., 2009). Además, se usan diferentes fluidos de inyección (aguas residuales con baja viscosidad) mezclados con algún material apuntalante (Dusseault y McLennan, 2011), constituidos por partículas mezcladas con el fluido de fracturamiento para mantener las fracturas abiertas; además de los granos de arena que aparecen naturalmente. También se pueden utilizar apuntalantes artificiales o de diseño especial, como arena cubierta con resina o materiales cerámicos de alta resistencia como la bauxita sinterizada (Schlumberger, 2019). Aunque la idea básica del fracturamiento de garantizar la conectividad dentro de la red de fracturas tiende a ser la misma en todas las industrias que lo utilizan (Adams y Rowe, 2013), y suele aplicarse en ambientes profundos a varios kilómetros de la superficie (Tester et al., 2006; Healy, 2012; Priestley, 2018), el objetivo de extracción es diferente: por un lado, en geotermia, se busca extraer el calor almacenado en las rocas que es un recurso renovable, y por otro, en la industria de hidrocarburos, el gas de esquisto atrapado en la roca, lo cual es un recurso no renovable (Sun et al., 2017).

\section{Fluido de fracturación}

En el caso de la extracción de gas, el fluido de fracturación que se utiliza necesita un componente que impida que la fractura se cierre completamente una vez terminado el bombeo, y que garantice suficiente conductividad en el canal recién creado (Al-Muntasheri, 2014). Este material se conoce como apuntalante o proppant en inglés (Montgomery y Smith, 2010), y debe poseer características como resistencia a los esfuerzos y a la corrosión, gravedad específica pequeña, entre otras, para asegurar que las fracturas permanezcan abiertas (de Campos et al., 2018).

Usualmente, en los EGS se realiza la estimulación inyectando agua sin apuntalante (Shiozawa y McClure, 2014), debido a que éstos se degradan en los sistemas geotérmicos que se caracterizan por fluidos químicamente agresivos (McClure y Horne, 2014). Aunque los apuntalantes no han sido utilizados ampliamente en proyectos EGS, existen casos donde se han empleado: el primer experimento EGS con apuntalante fue una pequeña inyección en 1974 
en Fenton Hill (Brown et al., 2012), mientras que otros experimentos fueron ejecutados en el proyecto Rosemanowes en 1989 (Bennett y Baker, 1989) y en el proyecto Groß Schönebeck (Zimmermann et al., 2010).

Algunos estudios sobre la estabilidad química de los apuntalantes bajo diferentes condiciones geotérmicas han demostrado que estos corren el riesgo de disolución en los sistemas geotérmicos, pero que esto no está relacionado con cambios en la resistencia de los mismos (Brinton et al., 2011). Además, otros trabajos sugieren que, al ser recubierto por materiales específicos, el apuntalante puede ser mucho más resistente a la degradación química y a alta temperatura (Raysoni y Weaver, 2013).

Los fluidos de fracturación para la extracción de gas contienen diferentes aditivos (Broderick et al., 2011) como: apuntalantes, ácidos, biocidas, estabilizadores de arcilla, inhibidores de corrosión, reductores de fricción, agentes gelificantes, entre otros (Meiners et al., 2013). Aunque existe una lista de sustancias químicas que entran en la composición de los fluidos de fracturación, no se proporciona un conjunto completo de datos debido a la privacidad de las empresas que los usan (Sutra et al., 2017). Algunas de estas sustancias han sido consideradas tóxicas (Waxman et al., 2011), incluidos elementos carcinógenos, productos químicos regulados por el agua potable segura y contaminantes atmosféricos peligrosos.

\section{Impactos asociados}

Con el desarrollo de las técnicas para la extracción de recursos subterráneos, los impactos ambientales potenciales necesitan ser analizados para garantizar el bienestar económico y de la sociedad, así como la protección de los recursos naturales. Por esta razón, es importante evaluar el riesgo de contaminación, así como los efectos adversos para la salud humana asociados con el proceso de fracturación hidráulica. En general, para reducir todo tipo de impacto, los sitios elegidos para implementar este proceso deberían ubicarse en una zona con una densidad de población relativamente pequeña (Meier et al, 2015). Eso fue destacado por la experiencia de Basilea en Suiza, cuyo proyecto fue desarrollado en un área poblada. Al contrario, por ejemplo, el sitio experimental de Fallon Forge se ubica $16 \mathrm{~km}$ al norte de Milford, la ciudad más cercana, y $350 \mathrm{~km}$ al sur de Salt Lake City; el área del proyecto es rural y abarca aproximadamente $25 \mathrm{~km}^{2}$. En Fenton Hill, de manera similar, los trabajos de investigación fueron realizados en un área ubicada a $56 \mathrm{~km}$ de la ciudad de Los Alamos, lejos de centros urbanos. La ubicación de Paralana en Australia es también aislada de áreas urbanas, cerca de una mina de uranio. Sin embargo, Soultz-Sous-Forêts indica que, con sus 3000 habitantes aproximadamente, no ha tenido problemas de oposición ya que parece ser un caso en el que el proyecto estaba bien arraigado en la historia del territorio y cumplieron con las expectativas de las comunidades (Chavot et al., 2018).

\section{Fracturación hidráulica (Fracking)}

El riesgo potencial de contaminación para el agua proviene de la extracción del gas (Stuart, 2012), del fluido inyectado (Zhang y Yang, 2015) y del agua de retorno liberada durante la extracción (Vengosh et al., 2013). Los productos químicos de fracking incluyen ácido clorhídrico, poliacrilamida, aceite mineral, isopropanol, potasio, cloruro y etilenglicol y bajas concentraciones de tampones de $\mathrm{pH}$, inhibidores de la corrosión, biocidas y agentes gelificantes (Stuart, 2012), entre otros aditivos que no se mencionan públicamente (Sutra et al., 2017) y que pueden causar contaminación en los cuerpos de agua y pueden ser perjudiciales para la salud.

Los grandes volúmenes de agua requeridos varían ampliamente y se estiman entre $10.000-20.000 \mathrm{~m}^{3}$ por pozo (EGEC, 2013) y pueden ejercer presión sobre los recursos de agua con impactos en otros usos y ecosistemas dependientes de esta (Broderick et al., 2011).

El fracking libera gas directamente a la atmósfera (Weinhold, 2012), tanto dióxido de carbono $\left(\mathrm{CO}_{2}\right)$, como metano $\left(\mathrm{CH}_{4}\right)$ (Bista et al., 2017), que repercuten en la cantidad de gases de efecto invernadero y por ende en el cambio climático (Howarth et al., 2011).

Los eventos sísmicos constituyen otro problema relacionado con el fracking (Johnston et al., 2016) y están asociados a la eliminación de las aguas residuales de la estimulación y de la salmuera por inyección en pozos profundos (Ellsworth, 2013). Por lo general, estos eventos no son detectables, a excepción de instrumentos sensibles, aunque en ocasiones se han producidos eventos sentidos por la población (Khyade, 2016). 


\section{Sistemas Geotérmicos Mejorados (EGS)}

En el caso de la geotermia, la generación de energía presenta ventajas frente a otros tipos de recursos, entre las cuales estan las bajas emisiones de gases de efecto invernadero y la pequeña ocupación de territorio. Los fluidos geotérmicos pueden contener minerales disueltos como boro, mercurio y arsénico, que pueden llegar a ser dañinos para la salud, la flora, la fauna y podrían llegar a contaminar las aguas (DiPippo, 2012) si no hay un sistema apropiado de manejo.

En el caso específico de los sistemas EGS, la sismicidad inducida causada por la estimulación es otro impacto que debe ser mitigado, así como la utilización del agua para la estimulación hidráulica (Clark et al., 2011) que puede provenir de los mismos fluidos geotérmicos, lo que representa poco o ningún impacto en las fuentes locales de agua dulce (Kagel et al., 2007). Eventos sísmicos se pueden producir también en los campos geotérmicos tradicionales (no EGS) por la operación de pozos de reinyección (Zang et al., 2014), generalmente son de bajas magnitudes y no son percibidos por las comunidades aledañas, sin embargo, pueden afectar la opinión pública hasta el punto de causar la suspensión o cancelación de un proyecto geotérmico (Evans et al., 2012). Otro impacto que está asociado a los campos geotérmicos tradicionales es la subsidencia del suelo cuando la tasa de extracción del fluido geotérmico es superior a la reinyección o recarga del reservorio, esto se evidenció en el campo Wairakei, Nueva Zelanda, donde no se utilizó la reinyección y las tasas de subsidencia en una parte del campo fueron hasta de $0,48 \mathrm{~m}$ por año (Allis, 2000). En el caso de los EGS, el reservorio se mantiene bajo presión continua y la cantidad de fluido en la formación se mantiene constante durante la operación de la planta, por lo que no se esperan impactos de subsidencia para los sistemas EGS (Tester et al., 2006).

\section{Percepción social}

El uso de energías alternativas para la mitigación del cambio climático ha encontrado, en algunas ocasiones, oposición debido a aspectos sociales, económicos, ambientales, tecnológicos y científicos (Kępińska y Kasztelewicz, 2015). Los impactos al medio natural, la falta de participación de las comunidades, los asuntos financieros y las dificultades relacionadas con la ubicación de los proyectos han generado resistencia en la aceptación de las técnicas empleadas, como es el caso de la energía nuclear, los sistemas eólicos (Hall et al., 2013), la tecnología de captura y almacenamiento de carbono (Van Noorden, 2010) y los proyectos geotérmicos (Reith et al., 2013).

La tecnología geotérmica todavía se considera emergente para la generación de energía. En Australia, la mayoría de los participantes en una encuesta estuvieron de acuerdo con la implementación de sistemas EGS para generación de energía geotérmica, sin embargo, presentan preocupaciones sobre los riesgos potenciales que podría ocasionar y se prefiere que esta generación se despliegue fuera de las comunidades (Carr-Cornish y Romanach, 2014). En la Península Biga, Turquía, los recursos de energía geotérmica tienen un potencial significativo para diferentes usos, sin embargo, existe una preocupación ya que indican que el conocimiento sobre los efectos ambientales es insuficiente (Çetiner et al., 2016). En países europeos (Hungría, Italia, Macedonia, Polonia, Rumania, Serbia y Eslovaquia), la aceptación pública de las energías renovables y geotérmica prevalece y es evaluada como aceptable o alta (Kępińska y Kasztelewicz, 2015).

En Colombia se desarrolló una encuesta donde se realizaban preguntas relacionadas con las energías renovables incluyendo la geotérmica, los impactos potenciales asociado al desarrollo de este tipo de proyectos y la estimulación hidráulica como técnica a la que se recurre para garantizar la viabilidad de algunos de los proyectos geotérmicos (Ramírez et al., 2017). En los resultados se evidenció que las energías renovables son aceptadas, consideradas como fuentes alternativas a los combustibles fósiles, permitiendo mitigar emisiones de gases contaminantes, sin embargo, no son vistas como prioridad en el país. Además, se observó que la mención y explicación del término fracking cambia la percepción de las personas con respecto a la implementación de este tipo de proyectos (Ramírez et al., 2017). Sin embargo, los sistemas geotérmicos colombianos de interés son de tipo hidrotermal, así que no están planeados sistemas EGS. De hecho, en cuanto al recurso geotérmico, Colombia cuenta con una posición geográfica privilegiada y una geología favorable, dado que parte del territorio se encuentra ubicado en el Cinturón de Fuego del Pacífico, zona donde el gradiente geotérmico es anómalamente alto (Marzolf, 2014). Se han detectado varios puntos con actividad para el desarrollo geotérmico, entre ellos se encuentra el Nevado del Ruiz en la zona de la Nereidas en Caldas, el Azufral de Tuquerres en el departamento de Nariño, Proyecto Geotérmico Binacional TufiñoChiles-Cerro Negro, y Paipa-Iza en el departamento de Boyacá (Arias y Acevedo, 2017). En todas estas 
regiones, se explotarían potenciales reservorios geotérmicos, sin necesidad de implementar sistemas EGS.

La aceptación de las comunidades donde se podría desarrollar un proyecto geotérmico es de vital importancia para su ejecución exitosa. Para esto es necesario realizar procesos de aprendizaje y participación ciudadana, procesos de socialización y sensibilización a las comunidades ubicadas en el área de influencia.

\section{CONCLUSIONES}

Los EGS han avanzado mucho desde sus inicios en los años 70 en Fenton Hill. Este primer proyecto, dio paso a la creación y réplica de la técnica en otros entornos geológicos como Soultz-Sous-Forêts en Francia, Basilea en Suiza, Groß Schönebeck en Alemania, Paralana en Australia, Ogachi en Japón, Rosemanawes en Reino Unido, New Berry y Fallon Forge en Estados Unidos. Los estudios de campo realizados en estos países durante varias décadas, han demostrado que la técnica es factible en términos de producción de energía geotérmica a través de regiones de roca estimulada a profundidades entre de 3000 a $5000 \mathrm{~m}$.

La mayoría de los EGS suponen que la estimulación se produce principalmente por deslizamiento inducido en las fracturas preexistentes (estimulación por cizallamiento puro), pero se ha observado que la estimulación puede ocurrir a través de una mezcla de mecanismos, deslizamiento de fracturas preexistentes y propagación de nuevas fracturas, es decir, se da estimulación mixta. En estos sistemas la composición de los fluidos usados durante la estimulación hidráulica no está definida claramente, a menudo es denominada como agua o fluido, dejando algunas dudas sobre el posible uso de aditivos.

El aprovechamiento de este tipo de energía tiene múltiples ventajas ya que es una energía limpia que permite la generación de electricidad de forma continua y confiable a través de beneficios que incluyen: una menor dependencia en los combustibles fósiles, uso diversificado de la energía y pocas emisiones de gases de efecto invernadero contribuyendo significativamente a la mitigación del cambio climático. Sin embargo, todo tipo de generación de energía genera un cierto impacto sobre el ambiente, que debe ser analizado previamente para ser mitigado.
El empleo de los EGS permite aumentar la viabilidad de los proyectos de explotación de recursos geotérmicos debido al incremento de la permeabilidad del reservorio, mediante la estimulación y creación de redes de fracturas.

\section{AGRADECIMIENTOS}

Los autores agradecen al proyecto internacional de investigación IGCP636: Unifying international research forces to unlock and strengthen geothermal exploitation of the Americas and Europe, del programa International Geosciences Programme (IGCP), apoyado por la UNESCO y IUGS (International Union of Geological Sciences).

\section{REFERENCIAS}

Adams, J., and Rowe, C. (2013). Differentiating applications of hydraulic fracturing. ISRM International Conference for Effective and Sustainable Hydraulic Fracturing, Brisbane, Australia.

Allis, R.G. (2000). Review of subsidence at Wairakei field, New Zealand. Geothermics, 29(4-5), 455478. doi: 10.1016/S0375-6505(00)00016-X.

Al-Muntasheri, G.A. (2014). A critical review of hydraulic-fracturing fluids for moderate-to ultralow-permeability formations over the last decade. SPE Production and Operations, 29(4), 243-260. doi: 10.2118/169552-PA.

Arias, G., y Acevedo, A.M. (2017). Estado actual de la producción de energía geotérmica en Colombia. Tesis. Universidad Nacional Abierta y a Distancia, Manizales, Colombia.

Ayling, B., Blankenship, D., Sullivan, P., Kennedy, M., Majer, E.L., Villavert, M., Sonnenthal, E., Tang, J., Dobson, P., Hinz, N., Faulds, J., Hammond, W., Mlawsky, E., Blake, K., Tiedeman, A., Sabin, A., Lazaro, M., Akerley, J., Nordquist, J., Sophy, M., Siler, D.L. , Kaven, J.O., Phelps, G., Hickman, S., Glen, J., Williams, C., Robertson-Tait, A., Hackett, L., Pettitt, W., Riahi A., Blanksma, D., Damjanac, B., Hazzard, J., Eneva, M., Witter, J.B., Queen, J., and Fortuna, M. (2018). Phase 2 Update for the Fallon FORGE Site, Nevada, 
USA. 43rd Workshop on Geothermal Reservoir Engineering. Stanford, California.

Barbier, E. (2002). Geothermal energy technology and current status: an overview. Renewable and Sustainable Energy Reviews, 6(1-2), 3-65. doi: 10.1016/S1364-0321(02)00002-3.

Bendall, B., Hogarth, R., Holl, H., McMahon, A., Larking, A., and Reid, P. (2014). Australian experiences in EGS permeability enhancement - A review of 3 case studies. 39th Workshop on Geothermal Reservoir Engineering. Stanford, California, USA.

Bennett, T.S., and Barker, K. (1989). Operational aspects of placing proppant in a naturally fractured geothermal reservoir. Geothermal Resources Council Transactions, 13, 359-365.

Bertani, R. (2012). Geothermal power generation in the world 2005-2010 update report. Geothermics, 41, 1-29. doi: 10.1016/j.geothermics.2011.10.001.

Bista, S., Jennings, P., and Anda, M. (2017). Cradle to grave GHG emissions analysis of shale gas hydraulic fracking in Western Australia. Renewable Energy Environmental Sustainability, 2(45), 1-6. doi: 10.1051/rees/2017014.

Blankenship, D., Kennedy, M., Majer, E.L., Hinz, N., Faulds, J., Ayling B., Blake, K., Tiedeman, A., Sabin, A., Lazaro, M., Akerley, J., Siler, D., Kaven, J.O., Phelps, G., Hickman, S., Glen, J., Williams, C., Robertson-Tait, A., Hackett, L., and Pettitt, W. (2017). Proposed Fallon FORGE Site: Phase 2 Update. 42nd Workshop on Geothermal Reservoir Engineering. Stanford, California, USA.

Blöcher, G., Cacace, M., Reinsch, T., and Watanabe, N. (2015). Evaluation of three exploitation concepts for a deep geothermal system in the North German Basin. Computers and Geosciences, 82, 120-129. doi: 10.1016/j.cageo.2015.06.005.

BP. (2019). BP Statistical Review of World Energy, 68th edition.

Brace, W.F. (1980). Permeability of crystalline and argillaceous rocks. International Journal of Rock Mechanics and Mining Sciences \& Geomechanics Abstracts, 17(5), 241-251. doi: 10.1016/01489062(80)90807-4.
Brinton, D., McLin, K., and Moore, J. (2011). The chemical stability of bauxite and quartz sand proppants under geothermal conditions. 36th Workshop on Geothermal Reservoir Engineering. Stanford, California, USA.

Broderick, J., Wood, R., Gilbert, P., Sharmina, M., Anderson, K., Footitt, A., Glynn, S., and Nicholls, F. (2011). Shale gas: an updated assessment of environmental and climate change impacts. Tyndall Centre for Climate Change Research. University of Manchester.

Brown, D.W. (2009). Hot dry rock geothermal energy: important lessons from Fenton Hill. 34th Workshop on Geothermal Reservoir Engineering. Stanford, California, USA.

Brown, D.W., Duchane, D.V., Heiken, G., and Hriscu, V.T. (2012). Mining the Earth's heat: Hot dry rock geothermal energy. Berlin: Springer.

Burton Jr, G.A., Basu, N., Ellis, B.R., Kapo, K.E., Entrekin, S., and Nadelhoffer, K. (2014). Hydraulic "Fracking": are surface water impacts an ecological concern?. Environmental Toxicology and Chemistry, 33(8), 1679-1689. doi: 10.1002/ etc. 2619 .

Carr-Cornish, S., and Romanach, L. (2014). Differences in public perceptions of geothermal energy technology in Australia. Energies, 7(3), 1555-1575. doi: 10.3390/en7031555.

Çetiner, Z.S., Ertekin, C., and Gültay, B. (2016). Initial assessment of public perception and acceptance of geothermal energy applications in Çanakkale, NW Turkey. Energy Procedia, 97, 194-201. doi: 10.1016/j.egypro.2016.10.052.

Chamorro-Camazón, C. (2009). Energía eléctrica a partir de recursos geotérmicos. Estado actual y perspectivas a nivel mundial. Dyna, 84(1), 44-51.

Chavot, P., Heimlich, C., Masseran, A., Serrano, Y., Zoungrana, J., and Bodin, C. (2018). Social shaping of deep geothermal projects in Alsace: politics, stakeholder attitudes and local democracy. Geothermal Energy, 6(26). doi: 10.1186/s40517018-0111-6.

Cladouhos, T.T., Petty, S., Bonneville, A., Schultz, A., and Sorlie, C.F. (2018). Super Hot EGS and the 
Newberry Deep Drilling Project. 43rd Workshop on Geothermal Reservoir Engineering. Stanford, California, USA.

Clark, C., Wang, M., Vyas, A., and Gasper, J. (2009). Life cycle approach to understanding impacts of EGS. Geothermal Resources Council Transactions, 33, 311-314.

Clark, C., Harto, C., Sullivan, J., and Wang, M. (2011). Water use in the development and operation of geothermal power plants. Argonne National Laboratory. 72 .

Conde-Álvarez, C., y Saldaña-Zorrilla, S.O. (2007). Cambio climático en América Latina y el Caribe: Impactos, vulnerabilidad y adaptación. Revista Ambiente y Desarrollo, 23(2), 23-30.

Cuenot, N., Faucher, J.P., Fritsch, D., Genter, A., and Szablinski, D. (2008). The European EGS project at Soultz-sous-Forêts: From extensive exploration to power production. 2008 IEEE Power and Energy Society General Meeting - Conversion and Delivery of Electrical Energy in the 21st Century. Pittsburgh, USA.

Darnet, M., Marquis, G., and Sailhac, P. (2006). Hydraulic stimulation of geothermal reservoirs: fluid flow, electric potential and microseismicity relationships. Geophysical Journal International, 166(1), 438-444. doi: 10.1111/j.1365246X.2006.03026.x.

de Campos, V.P.P., Sansone, E.C., and Silva, G.F.B.L. (2018). Hydraulic fracturing proppants. Cerâmica, 64(370), 219-229. doi: 10.1590/0366-69132018643702219.

DECC. (2013). Deep Geothermal Review Study Final Report. Department of Energy and Climate Change.

Dincer, I. (2000). Renewable energy and sustainable development: a crucial review. Renewable and Sustainable Energy Reviews, 4(2), 157-175. doi: 10.1016/S1364-0321(99)00011-8.

DiPippo, R. (2012). Geothermal power plants: Principles, applications, case studies and environmental impacts. Massachusetts: University of Massachusetts Dartmouth.
Dubois, M., Ayt Ougougdal, M., Meere, P., Royer, J.J., Boiron, M.C., and Cathelineau, M. (1996). Temperature of paleo to modern self-sealing within a continental rift basin: the fluid inclusion data (Soultz-sous-Forêts, Rhine graben, France). European Journal of Mineralogy, 8(5), 10651080. doi: $10.1127 /$ ejm/8/5/1065.

Dusseault, M., and McLennan, J. (2011). Massive multistage hydraulic fracturing: Where are we? 45th US Rock Mechanics/Geomechanics Symposium, San Francisco, California, USA.

Ellsworth, W.L. (2013). Injection-induced earthquakes. Science, 341(6142), 1-7 doi: 10.1126/science. 1225942 .

Elum, Z.A., and Momodu, A.S. (2017). Climate change mitigation and renewable energy for sustainable development in Nigeria: A discourse approach. Renewable and Sustainable Energy Reviews, 76, 72-80. doi: 10.1016/j.rser.2017.03.040.

EGEC. (2013). Factsheet on Enhanced Geothermal Systems (EGS): Why it's different to shale gas. European Geothermal Energy Council.

ENGINE. (2008). Geothermal lighthouse projects in Europe. Information gathered during the ENGINE co-ordination action (ENhanced Geothermal Innovative Network for Europe).

Evans, K.F., Zappone, A., Kraft, T., Deichmann, N., and Moia, F. (2012). A survey of the induced seismic responses to fluid injection in geothermal and $\mathrm{CO}_{2}$ reservoirs in Europe. Geothermics, 41, 30-54. doi: 10.1016/j.geothermics.2011.08.002.

Faulds, J.E., Blankenship, D., Hinz, N.H., Sabin, A., Nordquist, J., Hickman, S., Glen, J., Kennedy, M., Siler, D.L., Robertson-Tait, A., Williams, C., Drakos, P., and Calvin, W. (2015). Geologic setting of the proposed Fallon Forge Site, Nevada: Suitability for EGS research and development. Geothermal Resources Council Transactions, 39, 293-302.

Genter, A., Evans, K., Cuenot, N., Fritsch, D., and Sanjuan, B. (2010). Contribution of the exploration of deep crystalline fractured reservoir of Soultz to the knowledge of Enhanced Geothermal Systems (EGS). Comptes Rendus Geoscience, 342(7-8), 502-516. doi: 10.1016/j.crte.2010.01.006. 
Guo, C., Wei, M., and Lui, H. (2018). Study of gas production from shale reservoirs with multi-stage hydraulic fracturing horizontal well considering multiple transport mechanisms. PLOS ONE, 13(1), 1-21. doi: 10.1371/journal.pone.0188480.

Gurney, K.R., Mendoza, D.L., Zhou, Y., Fischer, M.L., Miller, C., Geethakumar, S., and de la Rue du Can, S. (2009). High resolution fossil fuel combustion $\mathrm{CO}_{2}$ emission fluxes for the United States. Environmental Science \& Technology, 43(14), 5535-5541. doi: 10.1021/es900806c.

Hall, N., Ashworth, P., and Devine-Wright, P. (2013). Societal acceptance of wind farms: Analysis of four common themes across Australian case studies. Energy Policy, 58, 200-208. doi: 10.1016/j.enpol.2013.03.009.

Häring, M.O., Schanz, U., Ladner, F., and Dyer, B.C. (2008). Characterisation of the Basel 1 enhanced geothermal system. Geothermics, 37(5), 469-495. doi: 10.1016/j.geothermics.2008.06.002.

Healy, D. (2012). Hydraulic Fracturing or 'Fracking': A Short Summary of Current Knowledge and Potential Environmental Impacts. Department of Geology \& Petroleum Geology, University of Aberdeen, United Kingdom.

Hochstein, M.P. (1988). Assessment and modelling of geothermal reservoirs (small utilization schemes). Geothermics, 17(1), 15-49. doi: 10.1016/03756505(88)90004-1.

Howarth, R.W., Santoro, R., and Ingraffea, A. (2011). Methane and the greenhouse-gas footprint of natural gas from shale formations. Climatic Change, 106(4), 679-690. doi: 10.1007/s10584011-0061-5.

Huenges, E., Saada, A., Brandt, W., Moeck, I., Holl, H.G., Zimmermann, G., Blöcher, G., Köhler, S., Legarth, B., and Tischner, T. (2006). Current status of the EGS gross schönebeck project: On the way to demonstrate sustainable brine production from deep sediments of the North German Basin. Geothermal Resources Council Transactions, 30, 341-346.

Johnston, J.E., Werder, E., and Sebastian, D. (2016). Wastewater disposal wells, fracking, and environmental injustice in Southern
Texas. American Journal of Public Health, 106(3), 550-556. doi: 10.2105/AJPH.2015.303000.

Kaieda, H. (2012). Ogachi EGS reservoir analysis. Geothermal Resources Council Transactions, 36, 487-492.

Kaieda, H., Ito, H., Kiho, K., Suzuki, K., Suenaga, H., and Shin, K. (2005). Review of the Ogachi HDR Project in Japan. World Geothermal Congress. Antalya, Turkey.

Kagel, A., Bates, D., and Gawell, K.A. (2007). A guide to geothermal energy and the environment. Geothermal Energy Association. Washington DC. 75 .

Kelkar, S., WoldeGabriel, G., and Rehfeldt, K. (2016). Lessons learned from the pioneering hot dry rock project at Fenton Hill, USA. Geothermics, 63, 5-14. doi: 10.1016/j.geothermics.2015.08.008.

Kępińska, B., and Kasztelewicz, A. (2015). Public perception of geothermal energy in selected European countries. World Geothermal Congress. Melbourne, Australia.

Khyade, V.B. (2016). Hydraulic fracturing; Environmental issue. World Scientific News, 40, 58-92.

King, J.P., Reid, P.W., and Bendall, B. (2009). Progress at the Paralana EGS Project in South Australia. Australian Geothermal Energy Conference. Brisbane, Australia.

Kraft, T., Mai, P.M., Wiemer, S., Deichmann, N., Ripperger, J., Kästli, P., Bachmann, C., Fäh, D., Wössner, J., and Giardini, D. (2009). Enhanced geothermal systems: mitigating risk in urban areas. Eos, Transactions American Geophysical Union, 90(32), 273-274. doi: 10.1029/2009EO320001.

Lacirignola, M., and Blanc, I. (2013). Environmental analysis of practical design options for enhanced geothermal systems (EGS) through life-cycle assessment. Renewable Energy, 50, 901-914. doi: 10.1016/j.renene.2012.08.005.

Lu, S.M. (2018). A global review of Enhanced Geothermal System (EGS). Renewable and Sustainable Energy Reviews, 81(2), 2902-2921. doi: 10.1016/j.rser.2017.06.097. 
Marzolf, N.C. (2014). Emprendimiento de la energía geotérmica en Colombia. Biblioteca Felipe Herrera del Banco Interamericano de Desarrollo 86.

Meier, P.M., Rodríguez, A.A., and Bethmann, F. (2015). Lessons learned from basel: New EGS projects in Switzerland using multistage stimulation and a probabilistic traffic light system for the reduction of seismic risk. World Geothermal Congress. Melbourne, Australia.

Meiners, H.G., Denneborg, M., Müller, F., Bergmann, A., Weber, F.A., Dopp, E., Hansen, C., and Schüth, C. (2013). Environmental impacts of fracking related to exploration and exploitation of unconventional natural gas deposits. Federal Ministry for the Environment, Nature Conservation and Nuclear Safety, 275.

Meng, Q., and Ashby, S. (2014). Distance: A critical aspect for environmental impact assessment of hydraulic fracking. The Extractive Industries and Society, 1(2), 124-126. doi: 10.1016/j. exis.2014.07.004.

McClure, M., and Horne, R. (2014). An investigation of stimulation mechanisms in Enhanced Geothermal Systems. International Journal of Rock Mechanics and Mining Sciences, 72, 242260. doi: 10.1016/j.ijrmms.2014.07.011.

Min, K.B., Park, S., and Zimmermann, G. (2015). Case Study on Groß Schönebeck EGS Project Research in Germany. Journal of Korean Society for Rock Mechanics, 25(4), 320-331. doi: 10.7474/ TUS.2015.25.4.320.

Montgomery, C.T., and Smith, M.B. (2010). Hydraulic fracturing: History of an enduring technology. Journal of Petroleum Technology, 62(12), 26-40. doi: 10.2118/1210-0026-JPT.

Olasolo, P. (2014). Análisis general sobre sistemas geotérmicos mejorados (EGS) para la generación de energía eléctrica. PhD. Tesis, Universidad de La Rioja, España.

Olasolo, P. Juárez, M.C., Morales, M.P., D'Amico, S., and Liarte, I.A. (2016). Enhanced Geothermal Systems (EGS): A review. Renewable and
Sustainable Energy Reviews, 56, 133-144. doi: 10.1016/j.rser.2015.11.031.

Popovska-Vasilevska, S. (2003). Drying of agricultural products with geothermal energy. International Summer School on Direct Application of Geothermal Energy, Izmir, Turkey.

Priestley, S. (2018). Shale gas and fracking. House of Commons Library.

Ramírez, E., López, J., Blessent, D., Raymond, J., Malo, M., y Balzán, D. (2017). Percepción social de la población rural en la zona de influencia del posible desarrollo geotérmico en el VNR. Reunión Nacional de Geotermia RENAG, Manizales, Colombia.

Raysoni, N., and Weaver, J. (2013). Long-term hydrothermal proppant performance. SPE Production and Operations, 28(4), 414-426. doi: 10.2118/150669-PA.

Reid, P., Messeiller, M., and Hasting, M. (2012). The Paralana Engineered Geothermal Project case history and results of the hydraulic fracture stimulation. Australian Geothermal Energy Conference. Australia.

Reiter, M., Weidman, C., Edwards, C.L., and Hartman, H. (1976). Subsurface Temperature Data in Jemez Mountains, New Mexico. New Mexico Bureau of Mines and Mineral Resources. A division of New Mexico Institute of mining \& Tecnology. $17 \mathrm{p}$.

Reith, S., Kölbel, T., Schlagermann, P., Pellizzone, A., and Allansdottir, A. (2013). Public acceptance of geothermal electricity production. Report on public acceptance, GEOELEC. $41 \mathrm{p}$.

REN21. (2019). Renewables 2019 Global Status Report. Renewable Energy Policy Network for the 21 st Century.

Robertson-Tait, A., Villavert, M., Kennedy, M., Blankenship, D., Sullivan, P., Tang, J., CamachoLopez, T., Nordquist, J., Akerley, J., Ayling, B., Majer, E., Faulds, J., and Roque-Rivera, R. (2018). Communications and outreach for public acceptance of complex technical projects: Experience from the Fallon FORGE Project. 43rd 
Workshop on Geothermal Reservoir Engineering, Stanford, California, USA.

Rongved, M. (2015). Hydraulic fracturing for enhanced geothermal systems. Master thesis, Norwegian University of Science and Technology, Norway.

Ruíz-Calvo, F. (2015). Análisis y modelado de una instalación geotérmica para climatización de un conjunto de oficinas. PhD Tesis, Universitat Politècnica de València, España.

Schill, E., Genter, A., Cuenot, N., and Kohl, T. (2017). Hydraulic performance history at the Soultz EGS reservoirs from stimulation and long-term circulation tests. Geothermics, 70, 110-124. doi: 10.1016/j.geothermics.2017.06.003.

Schindler, M., Baumgärtner, J., Gandy, T., Hauffe, P., Hettkamp, T., Menzel, H., Penzkofer, P., Teza, D., Tischner T., and Wahl, G. (2010). Successful hydraulic stimulation techniques for electric power production in the Upper Rhine Graben, Central Europe. World Geothermal Congress. Bali, Indonesia.

Schlumberger (2019). Agente de sustentación o apuntalante. Oilfield Glossary en Español. Consultado el 18 de noviembre de 2019. https:// www.glossary.oilfield.slb.com/es/Terms/p/ proppant.aspx

Shiozawa, S., and McClure, M. (2014). EGS designs with horizontal wells, multiple stages, and proppant. 39th Workshop on Geothermal Reservoir Engineering. Stanford, California, USA.

Siler, L., Hinz, N.H., Faulds, J.E., Ayling, B., Blake, K., Tiedeman, A., Sabin, A., Blankenship, D., Kennedy, M., Rhodes, G., Sophy, M.J., Glen, J.M.G., Phelps, G.A., Fortuna, M., Queen, J., and Witter, J.B. (2018). The geologic and structural framework of the Fallon FORGE site. 43rd Workshop on Geothermal Reservoir Engineering. Stanford, California, USA.

Stuart, M.E. (2012). Potential groundwater impact from exploitation of shale gas in the UK. Groundwater Science Program. British Geological Survey. Open report OR/12/00.
Sun, Z., Zhang, X., Xu, Y., Yao, J., Wang, H., Lv, S., Sun, Z., Huang, Y., Cai, M., and Huang, X. (2017). Numerical simulation of the heat extraction in EGS with thermal-hydraulic-mechanical coupling method based on discrete fractures model. Energy, 120, 20-33. doi: 10.1016/j.energy.2016.10.046.

Sutra, E., Spada, M., and Burgherr, P. (2017). Chemicals usage in stimulation processes for shale gas and deep geothermal systems: A comprehensive review and comparison. Renewable and Sustainable Energy Reviews, 77, 1-11. doi: 10.1016/j.rser.2017.03.108.

Tenzer, H. (2001). Development of hot dry rock technology. Geo Heat-Center Bulletin, 22(4), 1422.

Tester, J.W., Anderson, B.J., Batchelor, A.S., Blackwell, D.D., DiPippo, R., Drake, E.M., Garnish, J., Livesay, B., Moore, M.C., Nichols K., Petty, S., Toksöz, M.N. and Veatch, R.W. Jr. (2006). The future of geothermal energy. Impact of enhanced geothermal system (EGS) on the United States in 21 st Century. MIT-led interdisciplinary panel. $372 \mathrm{p}$.

Ucar, E., Berre, I., and Keilegavlen, E. (2018). Three-dimensional numerical modeling of shear stimulation of fractured reservoirs. Journal of Geophysical Research: Solid Earth, 123(5), 3891-3908. doi: 10.1029/2017JB015241.

U.S. Department of Energy. (2004). Geothermal Technologies Program: Enhanced Geothermal Systems. DOE/GO-102004-1958.

Van Noorden, R. (2010). Carbon sequestration: Buried trouble. Nature, News Feature, 463, 871-873. doi: 10.1038/463871a.

Vengosh, A., Warner, N., Jackson, R., and Darrah, T. (2013). The effects of shale gas exploration and hydraulic fracturing on the quality of water resources in the United States. Procedia Earth and Planetary Science, 7, 863-866. doi: 10.1016/j. proeps.2013.03.213.

Watanabe, N., Blöcher, G., Cacace, M., Held, S., and Kohl, T. (2017). Geoenergy Modeling III, Enhanced Geothermal Systems. Cham: Springer International Publishing. 
Waters, G., Dean, B., Downie, R., Kerrihard, K., Austbo, L., and McPherson, B. (2009). Simultaneous hydraulic fracturing of adjacent horizontal wells in the Woodford Shale. SPE Hydraulic Fracturing Technology Conference, The Woodlands, Texas, USA. doi: 10.2118/119635-MS.

Waxman, H.A., Markey, E.J., and DeGette, D. (2011). Chemicals used in hydraulic fracturing. United States House of Representatives Committee on Energy and Commerce Minority Staff.

Weinhold, B. (2012). The future of fracking: New rules target air emissions for cleaner natural gas production. Environmental Health Perspectives, 120(7), 272-279. doi: 10.1289/ehp.120-a272.

Wyss, R., and Rybach, L. (2010). Developing deep geothermal resources in Switzerland. World Geothermal Congress. Bali, Indonesia.

Xia, Y., Plummer, M., Mattson, E., Podgorney, R., and Ghassemi, A. (2017). Design, modeling and evaluation of a doublet heat extraction model in enhanced geothermal systems. Renewable Energy, 105, 232-247. doi: 10.1016/j.renene.2016.12.064.

Xu, T., Rose, P., Fayer, S., and Pruess, K. (2009). On modeling of chemical stimulation of an enhanced geothermal system using a high $\mathrm{pH}$ solution with chelating agent. Geofluids, 9(2), 167-177. doi: 10.1111/j.1468-8123.2009.00246.x.

Zang, A., Oye, V., Jousset, P., Deichmann, N., Gritto, R., McGarr, A., Majer, E., and Bruhn, D. (2014).
Analysis of induced seismicity in geothermal reservoirs - An overview. Geothermics, 52, 6-21. doi: 10.1016/j.geothermics.2014.06.005.

Zhang, D., and Yang, T. (2015). Environmental impacts of hydraulic fracturing in shale gas development in the United States. Petroleum Exploration and Development, 42(6), 876-883. doi: 10.1016/ S1876-3804(15)30085-9.

Zimmermann, G., Reinicke, A., Blöcher, G., Moeck, I., Kwiatek, G., Brandt, W., Regenspurg, S., Schulte, T., Saadat, A., and Huenges, E. (2010). Multiple fracture stimulation treatments to develop an Enhanced Geothermal System (EGS) - conceptual design and experimental results. World Geothermal Congress. Bali, Indonesia.

\begin{tabular}{c}
\hline María Alejandra Palacio-Villa. \\
ORCID: 0000-0001-5534-5856 \\
Daniela Blessent. \\
ORCID: 0000-0002-8347-381X \\
Jacqueline López-Sánchez. \\
ORCID: 0000-0003-0531-9478 \\
David Moreno. \\
ORCID: 0000-0003-0405-4308 \\
\hline \hline
\end{tabular}

Trabajo recibido: febrero 25 de 2019

Trabajo aceptado: noviembre 06 de 2019 